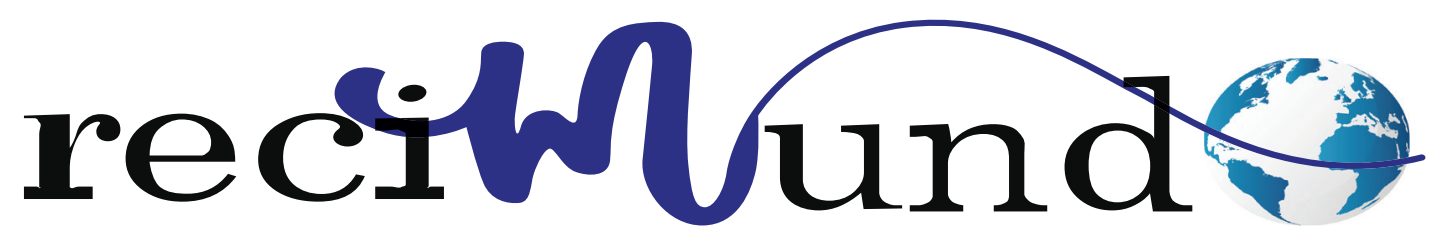

Revista Científica Mundo de la Investigación y el Conocimiento

DOI: 10.26820/recimundo/4.(2).mayo.2020.134-141

URL: http://recimundo.com/index.php/es/article/view/831

EDITORIAL: Saberes del Conocimiento

REVISTA: RECIMUNDO

ISSN: 2588-073X

TIPO DE INVESTIGACIÓN: Artículo de Revisión

Código UNESCO: 32 Ciencias Médicas; 3201 Ciencias Clínicas

PAGINAS: $134-141$

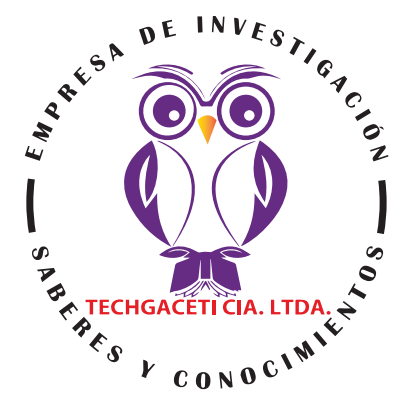

\title{
Pancreatectomía corporocaudal para el tratamiento de cistoadenoma seroso gigante. Reporte de caso
}

Corporocaudal pancreatectomy for the treatment of giant serous cystadenoma. Case report

Pancreatectomia corporocaudal para o tratamento de cistadenoma seroso gigante. Relato de caso

María Verónica Crespo Zamora'; Freddy Alberto Betancourt Bohórquez;

Paul Hamilton Capa Bohorquez;; Germán Giovanny Bravo Bohórquez ${ }^{4}$

RECIBIDO: 15/03/2020 ACEPTADO: 20/03/2020 PUBLICADO: 20/05/2020

1. Médico, Investigador Independiente; Guayaquil, Ecuador; veronicacrespoz29@outlook.com; (D) https://orcid. org/0000-0002-4098-7618

2. Médico, Investigador Independiente; Guayaquil, Ecuador; fsoruf@gmail.com; iD https://orcid.org/0000-00016327-6440

3. Médico, Investigador Independiente; Guayaquil, Ecuador; Insx27@hotmail.com; iD https://orcid.org/00000002-8152-6965

4. Médico, Investigador Independiente; Guayaquil, Ecuador; german_giova@hotmail.com; iD https://orcid. org/0000-0002-4945-3882

CORRESPONDENCIA

María Verónica Crespo Zamora

veronicacrespoz29@outlook.com

Guayaquil, Ecuador

() RECIMUNDO; Editorial Saberes del Conocimiento, 2020 


\section{RESUMEN}

Las neoplasias quísticas del páncreas son infrecuentes, constituyen el 15\% de todas las lesiones quísticas que se presentan, los pacientes son asintomáticos en su mayoría; sin embargo cuando aparece la sintomatología, ésta se caracteriza por dolor abdominal, náusea, vómito, dispepsia, melena, rara vez masa abdominal palpable, ictericia. Por lo que en la mayoría de los casos, el diagnóstico es incidental, se sugiere la utilización de diferentes métodos de exámenes complementarios entre los que destacan la TAC y la Resonancia magnética, el tratamiento de elección es quirúrgico y la técnica depende de la ubicación de la lesión quística. En ciertos casos se considera la Esplenectomìa por morbilidad posoperatoria. Se presenta un caso de cistoadenoma seroso pancreático gigante en una paciente de la cuarta edad, que se extiende de la cabeza al cuerpo del páncreas, se resuelve con pancreatectomìa corporocaudal.

Palabras clave: Páncreas, neoplasias quísticas, cistadenoma seroso, Pancreatectomía corporocaudal, tomografía, esplenectomía.

\section{ABSTRACT}

Cystic neoplasms of the pancreas are infrequent, constituting 15\% of all cystic lesions presented, patients are mostly asymptomatic; however, when symptomatology appears, it is characterized by abdominal pain, nausea, vomiting, dyspepsia, mane, rarely palpable abdominal mass, jaundice. As in most cases, the diagnosis is incidental, it is suggested the use of different methods of complementary examinations among which highlight the CT and MRI, The treatment of choice is surgical and the technique depends on the location of the cystic lesion. In certain cases, splenectomy is considered due to postoperative morbidity. A case of giant pancreatic serous cystoadenoma is presented in a patient of the fourth age, which extends from the head to the body of the pancreas, is resolved with corporeal pancreatectomy.

Keywords: Pancreas, cystic neoplasm, serous cistoadenoma, corporocaudal pancreatectomy, CT, splenectomy.

\section{RESUMO}

As neoplasias císticas do pâncreas são infreqüentes, constituindo 15\% de todas as lesões císticas apresentadas; os pacientes são na maioria assintomáticos; no entanto, quando a sintomatologia aparece, é caracterizada por dor abdominal, náusea, vômito, dispepsia, crina, massa abdominal raramente palpável, icterícia. Como na maioria dos casos, o diagnóstico é incidental, sugere-se o uso de diferentes métodos de exames complementares, dentre os quais destacam-se a TC e a RM. O tratamento de escolha é cirúrgico e a técnica depende da localização da lesão cística. Em certos casos, a esplenectomia é considerada devido à morbidade pós-operatória. Um caso de cistoadenoma seroso pancreático gigante é apresentado em um paciente da quarta idade, que se estende da cabeça ao corpo do pâncreas, e é resolvido com pancreatectomia corporal.

Palavras-chave: Pâncreas, neoplasia cística, cistoadenoma seroso, pancreatectomia corporocaudal, TC, esplenectomia. 


\section{Introducción}

Las neoplasias quísticas del páncreas conforman el $1 \%$ de todos los tumores primarios del páncreas y sólo el 15\% de las lesiones quísticas. ${ }^{3}$ Son tumores pocos comunes con gran variedad clínica, mayormente benignos; sin embargo, deben estudiarse para descartar malignidad, mayormente tienen buena respuesta al tratamiento quirúrgico. 2,1

La OMS clasificó los tumores quísticos más frecuentes: Cistoadenoma seroso (38\%), cistoadenoma mucinoso intraductal (30.6\%), tumores sólidos pseudopapilares $(11.9 \%)$ y neoplasias mucinosas $(9.7 \%){ }^{2}$ su distinción es de gran importancia para el manejo del tratamiento correcto. ${ }^{6,9}$

El cistoadenoma seroso del páncreas (SCA) es la neoplasia benigna más frecuente en mujeres entre la $5^{a}$ y $6^{a}$ década de vida con raras excepciones que degeneran a cistoadenocarcinoma seroso. ${ }^{2,9}$ Siendo de difícil diagnóstico clínico al ser su tamaño proporcional a la aparición de síntomas entre ellos: dolor abdominal, náusea, vómito, dispepsia, melena, rara vez masa abdominal palpable, ictericia, hipertensión portal, hemoperitoneo o pancreatitis. ${ }^{2,7}$ Apareciendo por compresión de estructuras vecinas. .,11 $^{4}$

Existen autores que para su manejo indican cirugía basado en cifras bajas de morbimortalidad versus otros los cuales exigen un mayor grado de certeza diagnóstica con la finalidad de disminuir al máximo la posibilidad de morbilidad innecesaria. ${ }^{10}$

Se recomienda la resección en caso de tumores quísticos serosos de un diámetro mayor a $4 \mathrm{~cm}$. Por otra parte, la observación de la lesión como parte del manejo sería posiblemente útil en los casos de lesiones ubicadas en la cabeza, en pacientes de edad y con mayor riesgo operatorio. ${ }^{10}$

Se presenta el caso de una paciente de sexo femenino con diagnóstico de SCA entre cuerpo y cabeza de páncreas a la que se le realiza pancreatectomía corporocaudal.

\section{Material y método}

Durante el periodo comprendido entre mayo a junio del 2009, se operó en el servicio de cirugía del Omni Hospital un caso de cistoadenoma seroso de páncreas. Se presenta caso clínico de una paciente de sexo femenino que acude hemodinámicamente inestable a emergencia con estudios de imágenes que demuestran un SCA entre cabeza y cuerpo de páncreas de $11 \mathrm{x}$ $11.8 \mathrm{~cm}$ de diámetro que dilata el conducto principal pancreático. Por la presencia de síntomas y localización de la lesión quística, se realiza pancreatectomía corporocaudal. Se confirmó el diagnóstico por estudio anatomopatológico.

\section{Caso clínico}

Paciente de sexo femenino con 45 años de edad, con antecedentes patológicos personales de hipertensión arterial (HTA), fue llevada a emergencia por cuadro clínico compatible con dolor abdominal intenso en hemiabdomen superior, posterior a una Endoscopía no determinada, acompañado de náuseas, vómitos y alza térmica de $40^{\circ} \mathrm{C}$.

Es ingresada a cuidados intermedios alerta, despierta, afebril con abdomen distendido y doloroso con signos relacionados a peritonismo. Se realiza exámenes de sangre destacando una leucocitosis 21.960 con neutrófilos $87 \%$ tratada con piperacilina tazobactam, posterior a tres días se normalizan los valores.

\section{Entre los estudios realizados se observó:}

TAC PELVIS Y ABDOMEN SIMPLE + CONTRASTE ORAL + IV: una lesión ocupante en cabeza del páncreas que se extiende a cuerpo pancreático con de $11 \times 11.8 \mathrm{~cm}$ con presencia de septos en su interior y dilatación del conducto principal pancreático 
distal (Figura 1,2,3)

COLANGIORESONANCIA: Lesión de aspecto quístico con tabiques en su interior lobulado, ubicado entre la cabeza y cuerpo del páncreas de aspecto quístico lobulado de 10.6 × $8.1 \mathrm{~cm}$, que provoca dilatación del conducto principal pancreático a nivel distal en cola pancreática.

La relación clínica más los estudios de imágenes diò el diagnóstico de tumoración quística en cabeza y cuerpo del pàncreas + sepsis abdominal por lo que se procede a realizar Pancreatectomìa corporocaudal, drenaje de quiste pancreático de gran tamaño de cabeza y cuerpo (800cc), colecistectomía, colocación de drenaje aspiratorio. Paciente egresa en posoperatorio, al momento ingresa a cuidados intensivos, $\mathrm{SaO} 2$ de 98\%.; presenta dos drenes abdominales de Blake, con poca producción hemática. Al primer día posoperatorio presenta irritabilidad, picos febriles, dolor abdominal, acompañados de Leucocitosis de 50.900 con neutrófilos de 95\%, enzimas hepáticas: Alt 2.356 y Ast 3,214.

Se realiza TAC de tórax, abdomen y pelvis; la cual muestra derrame pleural izquierdo, más colección abdominal en área del cuerpo pancreático extirpado. Se realiza Ecoendoscopìa para drenaje de colección peripancreàtica con Stent metálico más punción aspiración con aguja fina para obtención de muestra de estudio bacteriológico. Posteriormente se procede a realizar CPRE + Esfinterectomìa + Colocaciòn de Stent plástico pancreático.

Reporte del resultado de cultivo de líquido peripancreática aisló STENOTROPHOMONA MALTHOPHILIA, tratada con Minociclina y Levofloxacino.

Se realiza TAC de Abdomen y Pelvis con contraste IV: Focos de aspecto isquémico a nivel de hígado y bazo; vesícula ausente, colección peripancreática ya comentada con presencia de STENT de drenaje hacia estómago, alteración de la grasa mesentérica en forma difusa, secuelas quirúrgicas en pared abdominal anterior varices ùtero - ovárica izquierda.

Se realiza a los 15 días Ecoendoscopìa de control observándose: Colección 7cm aproximadamente, se decide no retirar Stent Hot Axios de $10 \mathrm{~mm}$.

A los 42 días de hospitalización se decide alta mèdica, con indicación de Ecoendoscopìa de control en 15 días, que se detalla en la sección de imágenes, figura 4. 


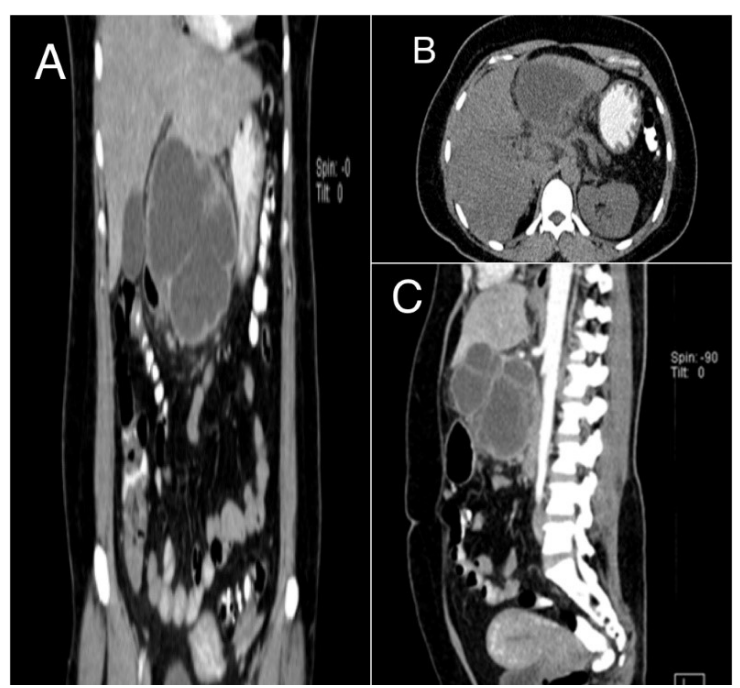

Imagen 1. Tomografía computarizada de pelvis y abdomen simple: A: tac abdomen y pelvis, muestra gran lesión ocupante en cabeza del páncreas que se extiende a cuerpo pancreático con de $11 \times 11.8 \mathrm{~cm}$ con presencia de septos en su interior y dilatación del conducto principal pancreático distal; B: corte coronal de abdomen simple C: corte sagital de tac abdomen simple, lesión lobulada, presencia de septos en su interior.

Fuente: Los autores

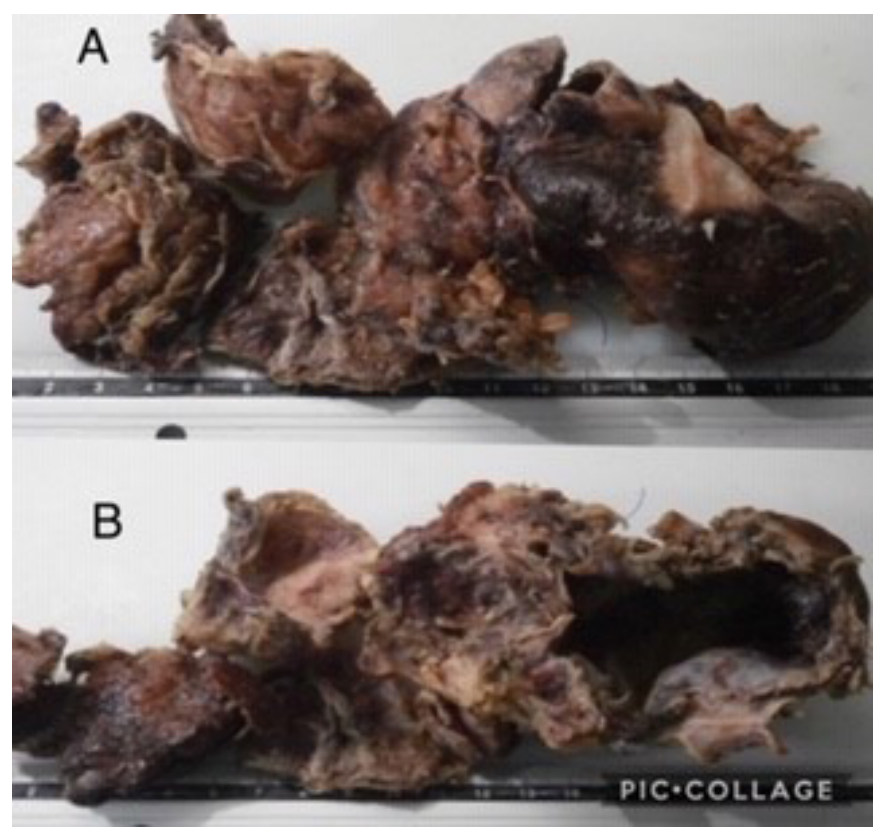

Imagen 2. Pared de quiste de páncreas. A: con un peso de 158 gramos y mide entre 12 y $6 \mathrm{cms}$. superficie parcialmente lisa, gris violácea con múltiples adherencias membranosas, amarillo blanquecinas. B: Al corte la pared mide entre 1 y $0,5 \mathrm{cms}$. superficie interna rugosa con material membranoso blanquecino adherido y áreas de apariencia hemorrágica, gris violáceo.

Fuente: Los autores 


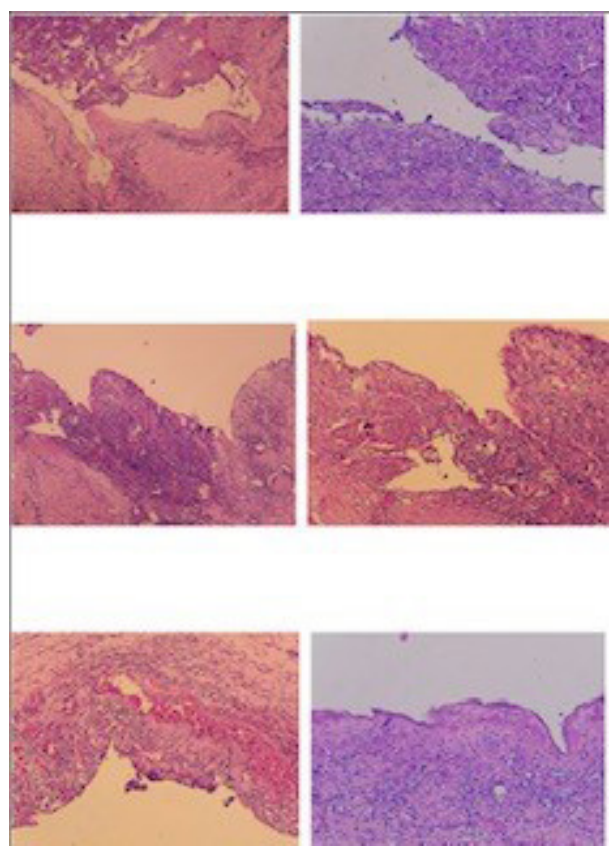

Imagen 3. Examen histopatológico, se observó la presencia de células inflamatorias con exudado de leucocitos polimorfonucleares neutrófilos y eosinòfilos; acompañado de mononucleares del tipo de linfocitos, células plasmáticas que infiltran un tejido fibroconectivo edematoso.

Fuente: Los autores

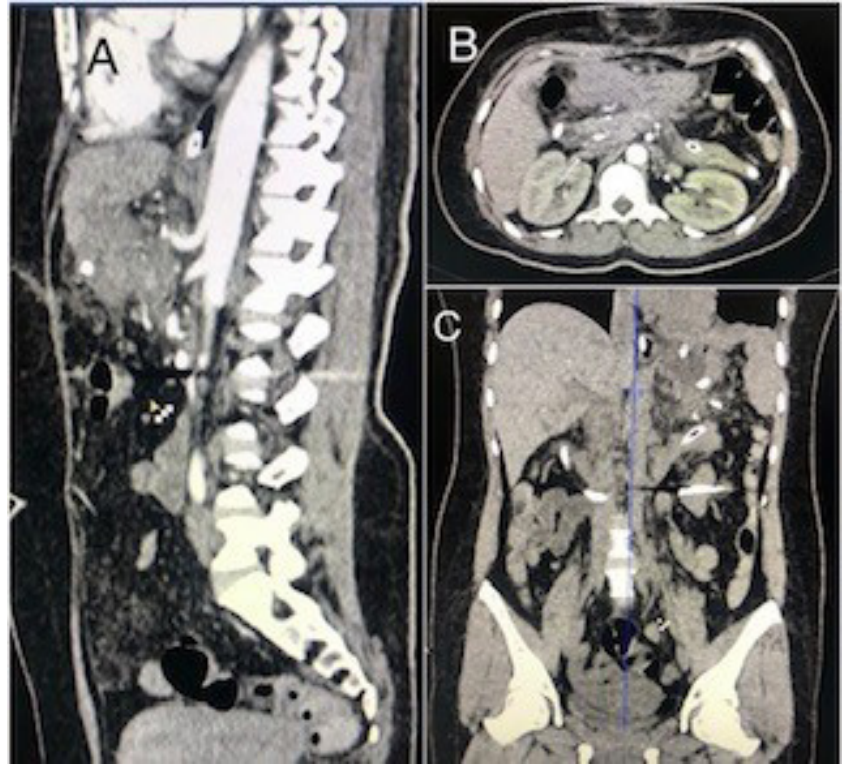

Imagen 4. Tomografía computarizada de pelvis y abdomen simple contrastada: A: corte sagital de tac abdomen simple, pequeña cantidad de líquido en fondo de saco. B: corte coronal; no adenopatías retroperitoneales. C: corte axial de tac abdomen y pelvis simple, focos de aspecto isquémico a nivel de hígado y bazo en menor cantidad y de menores dimensiones con respecto a estudio anterior. Cambios posquirúrgicos en cabeza de páncreas asociados a alteraciones de la grasa peripancreática sin presencia de colecciones observándose Stent de drenaje hacia estómago y Stent en conducto pancreático.

Fuente: Los autores 


\section{Discusión}

Los tumores quísticos del páncreas representan un reto al tener que dar un diagnostico según sus características antes de la intervención quirúrgica. ${ }^{6,12}$ EI SCA tiene una gran variedad morfológica, en la tomografía (TAC) se puede observar: Microquísticos (0.1 a $2 \mathrm{~cm}$ ) en forma de racimos de uva o una disposición en panal de abejas, los Macro/oligoquísticos (>2cm )son uniloculados o multiquístico y una variante solida de múltiples microquistes, en la TAC solo se puede distinguir la morfología del 60 al 70\% de los casos. ${ }^{2,4,5}$

Entre las diversas neoplasias quísticas el cistoadenoma de tipo mucinoso representa el 28 a 54\% de neoplasias malignas y muchas veces son difíciles de diferenciar en la TAC con los Macro/oligoquísticos del SCA. ${ }^{3,7}$

El uso de estudios de imágenes (tomografía computarizada, ultrasonografía, angiografía, y la Colangiopancreatografía endoscópica retrógrada) ayudan al descubrimiento de lesiones pancreáticas sin embargo estas técnicas tienen 25-50\% de error diagnóstico y se aconseja combinarla con citología mediante aspiración con aguja fina (BAAF).4,13

En general la resección quirúrgica completa es el tratamiento más indicado, tiene buenos resultados; sin embargo la principal complicación es el riesgo de fístula pancreática en $30-40 \%$ de pacientes.

La Esplenectomía concomitante se asocia a un aumento de morbilidad postoperatorio por una disminución circulatoria de anticuerpos, macrófagos y en el porcentaje de CD4 y linfocitos asociándose a un mayor número de infecciones. Sin embargo su preservación puede representar un alto riesgo de complicación, por lo que la conservación debe ser evaluada en cada caso. ${ }^{7}$

\section{Conclusión}

Los tumores quísticos representan un problema en el tratamiento de elección ya que este tipo de tumores tienen una sintomatología variada en relación a su tamaño, por lo que el tratamiento quirúrgico es la primera elección.

En el caso descrito se trató de un cistoadenoma seroso gigante que se resolvió con una pancreatectomìa corpocaudal, drenaje de quiste pancreático de gran tamaño de cabeza y cuerpo (800cc), colecistectomía y colocación de drenaje aspiratorio. Como complicación posoperatoria presentó una colección de 10 cms en área extirpada pancreática, que fue resuelta por medios endoscópicos descritos anteriormente, paciente fue dado de alta satisfactoriamente.

\section{Bibliografía}

1. Targarona, Javier, Garatea, Rafael, Romero, Cesar, Rosamedina, José Luís, Lora, Alfonso, Beltrán, Jorge, Rotta, César, Tapia, Pedro, \& Montoya, Eduardo. (2007). Tratamiento quirúrgico de los Cistoadenoma Serosos gigantes del páncreas: reporte de dos casos. Revista de Gastroenterología del Perú, 27(1), 85-90. Recuperado en 23 de octubre de 2019, de http://www. scielo.org.pe/scielo.php?script=sci_arttext\&pi$d=S 1022-51292007000100012 \&$ Ing=es\&tIng=es.

2. Venturelli F, Cárcamo C.(2007). Pancreatectomía corporocaudal con preservación esplénica. 21 : $31-37$

3. Farina, Miguel, Aguilera, Omar, Pederzoli, Rodrigo, \& Gómez, Diana. (2015). TRATAMIENTO LAPAROSCÓPICO DE CISTOADENOMA SEROSO DE COLA DE PÁNCREAS: REPORTE DE UN CASO: LAPAROSCOPIC TREATMENT OF SEROUS CYSTADENOMA IN TAIL OF PANCREAS: REPORT OF ONE CASE. Cirugía paraguaya, 39(1), 25-27. https://dx.doi.org/10.18004/sopaci.2015.junio.25-27

4. Domínguez I, Gómez-Sanz R, Vieiro-Medina, Et al. (2017). Central pancreatectomy for the treatment of a benign pancreatic lesion: case report and literature review. Revista Española de Enfermedades Digestivas, 109(3), 225-228. https://dx.doi. org/10.17235/reed.2017.4291/2016

5. Aristi Urista G, Güereña Elizalde A, Ramos de la 
Cruz R.(2002). Cistoadenoma seroso del páncreas ("adenoma microquístico"). Informe de siete casos. Rev. Chilena de Cirugía 65(1):15 - 22.

6. BUTTE B, NORERO M, DUARTE G.(2004).Tumores quísticos del páncreas. Rev. Chilena de Cirugía. 56(4): 3414-345

7. Álvarez L , Mejía J , Hoyos S.(2012).Neoplasias quísticas del páncreas. Rev Colomb Cir. 27:63-7

8. Zandalazine H, Oria A, Ocampo C.(2005).Complicaciones locales del cistoadenoma seroso de páncreas. Rev Arg Cirg.88(3-4):159-164

9. Yeo C. J., Matthews J. B., (2015) Cìrugìa del Tracto Alimentario de Shackelford. 7ma ed. Vol 2. Colombia: Amolca 2504 (1)

10. Xabier de Aretxabala, Iván Roa, Jorge León, Fernando Maluenda.(2008). Tumores quísticos del páncreas. Rev Méd Chile 2008; 136: 1188-1196 https://scielo.conicyt.cl/scielo.php?script=sci_arttext\&pid=S0034-98872008000900016
11. Mori E, Yuen K, Medrano H, et al. (2012). Manejo de los tumores quísticos pancreáticos en el hospital Alberto Sabogal Sologuren. Rev Gastroenterol Perú. 32 (2): 169-77.

12. Beane JD, Borrebach JD, Zureikat AH, et al. (2019). Cirugía pancreática óptima: ¿estamos progresando en América del Norte?. Ann Surg. 28 de octubre de 2019 doi: 10.1097 / SLA.0000000000003628

13. Guerrero García A, González-Huix F, Levy MJ, et al. (2019) .Terapia ablativa en lesiones quísticas pancreáticas. Gastroenterol Hepatol. 2019 enero; 42 (1): 43-50. doi: 10.1016 / j.gastrohep.2018.07.004

\section{CITAR ESTE ARTICULO:}

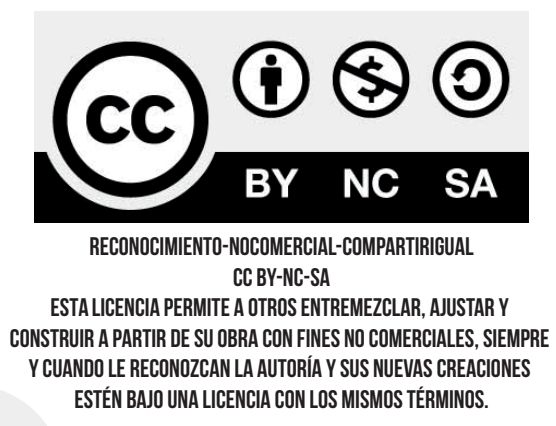

Crespo Zamora, M., Betancourt Bohórquez, F., Capa Bohorquez, P., \& Bravo Bohórquez, G. (2020). Pancreatectomía corporocaudal para el tratamiento de cistoadenoma seroso gigante. Reporte de caso. RECIMUNDO, 4(2), 130-137. doi:0.26820/recimundo/4.(2).mayo.2020.134-141 